\title{
An Indentation Method for Measuring Wear
}

\author{
By Samuel A. McKee
}

\begin{abstract}
This paper describes a method for accurately measuring the wear that takes place on the bearing surfaces of machinery. The method consists of making minute indentations in the wearing surface by means of a specially shaped diamond point. As material is worn from the surface, the dimensions of the marks change with the amount of material removed. Measurements of the dimensions of these marks before and after wear provide a means for determining the amount of wear that has taken place.

Apparatus is described for using this method for the measurement of wear occurring in the cylinders of aircraft engines. The results of tests with engines in operation indicate that the method is practicable. Curves are also given showing typical wear data obtained in engine cylinders.

The chief advantages of the method over the usual methods are (1) greater accuracy, (2) a precise indication as to where wear occurs, and (3) the measurements are not affected by growth, shrinkage or distortion of the machine element under test.
\end{abstract}

\section{Introduction}

One of the major difficulties in the determination of the wear of machinery has been the lack of a convenient method for measuring directly the amount of material removed. Among the methods that have been commonly used are the weighing of the parts before and after wear, the measurement of the dimensions of the parts before and after wear, and the determination of the amount of worn material found in the lubricant after operation.

In some cases, more especially with laboratory apparatus, the parts are of such size and shape that the wear may be determined with reasonable accuracy by weighing or by measuring the dimensions. In the normal operation of many machines, however, the amount of material worn off in a reasonable time is so small compared to the size of the part as to make it impracticable to determine the wear by loss in weight. The method of measuring changes in dimensions requires sensitive apparatus and painstaking technic to obtain the accuracy required. Even under ideal conditions both of these methods have some serious limitations. The weighing method gives a value for the total wear but does not indicate where the wear occurred. The method of measuring the changes in the dimensions of the part has this same limitation to a lesser degree in cases where wear occurs upon both surfaces that determine a given dimension. This method also does not differentiate between actual wear and other changes that may occur such as growth, shrinkage, or distortion of the part. The method of determining the amount of worn material in the lubricant presents a number of difficulties and usually is used only as a qualitative indication in conjunction with other data.

These limitations indicate the desirability of developing a method or methods of measuring wear that are independent of the size or mass of the part. The idea of the use of such a method is not new. For years engineers have used the appearance of the tool marks on a part as an approximate indication of the amount of wear. This practice suggested an investigation to determine the practicability of a method of measuring the amount of material worn off. This was done by applying to the working surfaces marks, of such a shape that a relatively small amount of wear would make a definite change in one or more readily measurable dimensions of the marks and 
hence provide a measurable indication of wear at the points where the marks are made.

The problem of particular interest in this investigation was the study of wear in radial aircraft engines, especially in the cylinders where the conditions are such that permanent distortion might occur during operation that could nullify, from the standpoint of measuring wear, the significance of even the most careful measurement. The apparatus therefore was designed primarily to locate and measure marks on the cylinder walls of a radial aircraft engine, although provision was also made for measuring the pistons.

\section{Method and Apparatus}

The choice of the particular type of mark used in this investigation was influenced by the fact that at the National Bureau of Standards there was already under development an indentation method for measuring the hardness of materials that utilized a mark that appeared to be readily adaptable for the measurement of wear. This hardness apparatus is known as the Knoop Indenter. ${ }^{1}$ The indentation is made by pressing into the surface the apex of a four-sided diamond pyramid having a rhombus-shaped base. The diamond used in these wear tests is of the same general shape as the Knoop diamond but differs in essential dimensions. Greatly enlarged views of a typical indentation are shown in figure 1 . The views on the left from top to bottom are the plan, longitudinal section, and transverse section respectively of a mark before wear has occurred, while those on the right are the respective views of the same mark after material has been removed from the surface. From this figure it will be noted that the shape of the mark remains the same, but the length decreases in proportion to the thickness of material removed. With the diamond used in these tests the ratio of depth of mark to length of long axis is about 1 to 35 , that is, a mark about 0.001 in. deep has a long axis of about 0.035 in.

Special apparatus was developed for making and measuring these marks. A cylinder or piston to be tested is placed in a special fixture, and marks at any desired position on the surface are applied to a predetermined depth by means of the diamond point, which is forced by mechanicai

\footnotetext{
${ }_{1}$ Frederick Knoop, Chauncy G. Peters, and Walter B. Emerson, J. Research NBS 23, 39 (1939) RP1220.
}
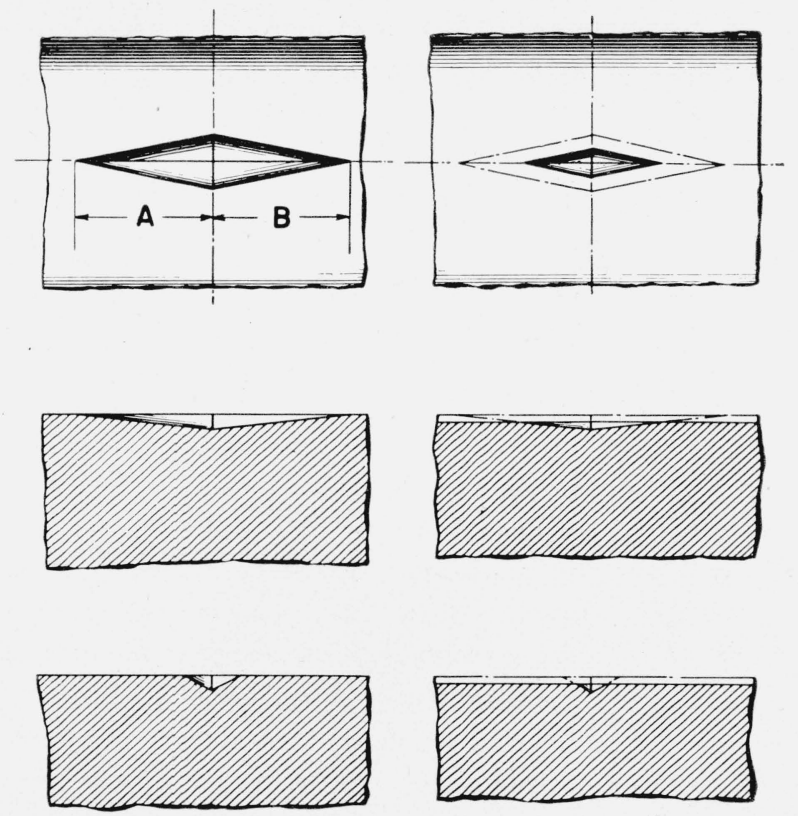

FIGURE 1.-Enlarged views of typical indentation.

pressure through suitable cams and levers into the surface of the part. A photograph of the apparatus for indenting the cylinders is shown in figure 2 and a drawing of this apparatus in figure 3 .

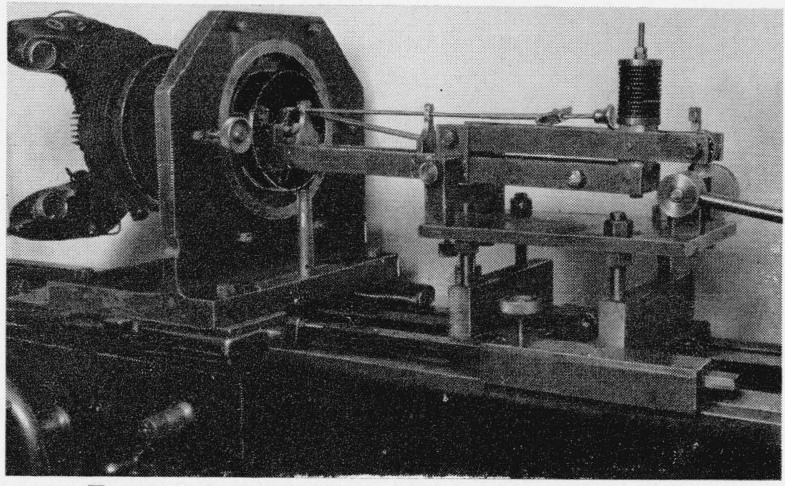

FiguRE 2.-Apparalus for indenting cylinders.

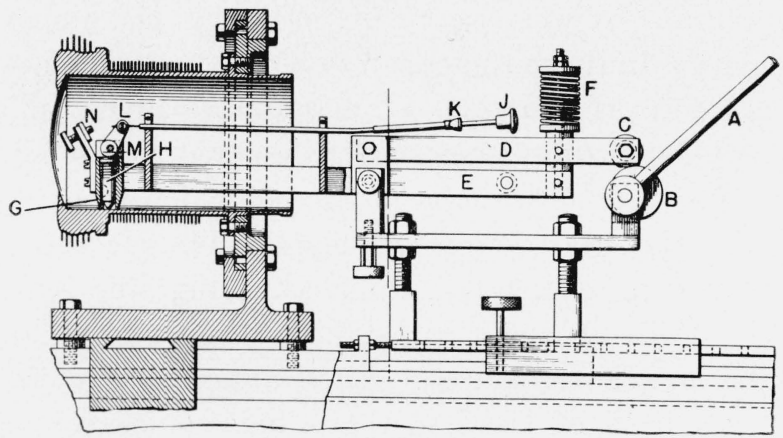

Figure 3.-Drawing of apparatus for indenting cylinders. 
In making an indentation, the cylinder is mounted in the fixture as shown in figure 3 . The cam lever, $A$, is moved by one hand of the operator to rotate the cam, $B$, in engagement with the roller, $C$, and raise lever, $D$. The upward movement of $D$ is communicated to lever, $E$, through the spring assembly, $F$. This depresses the indentation head, $G$, until it is clamped solidly against the surface of the cylinder by the force of spring, $F$. The indentation pin, $H$, carrying the diamond indentor is then depressed by the other hand of the operator by pushing the plunger, $J$, into its handgrip, $K$, thus moving the link, $L$, and actuating the indentation cam, $M$, on the indentation head. The cam contacting with the head of the indentation pin forces it downward and impresses the diamond indentor into the surface of the cylinder, the stop, $N$, regulating the depth of the mark. On removal of both hande the indentation head and pin return to their original position. The apparatus is arranged for the use of both hands in order to prevent the operator moving the cylinder relative to the indenter until the diamond is in a safe position.

The viewing and measuring apparatus consists of a conventional microscope and eye piece scale except that the tube is lengthened and two rightangle prisms inserted to permit use as a periscope to view the inside of the cylinders. A photograph of this apparatus as used in measuring a mark on a piston is shown in figure 4 .

A particular advantage of the use of this

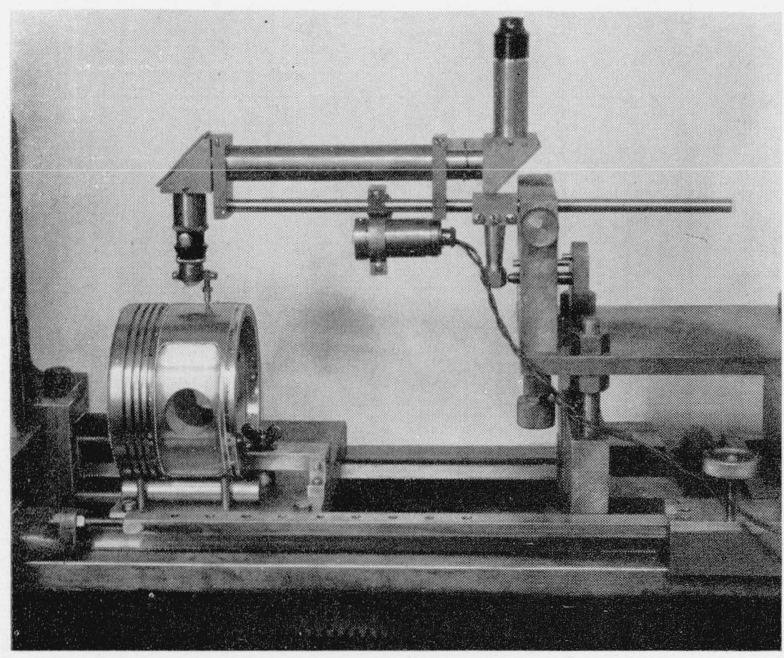

Figure 4.-Apparatus for viewing and measuring indentations. method and apparatus is that it provides an indication of wear only, while the usual measurements of changes in diameters of the pistons and cylinders do not differentiate between wear and such distortion as may occur during a test. A further advantage is that it indicates the wear at a particular point on the surface, whereas the measurements of diameter involve changes at two points, and usually there is no practical method of determining how much of the wear occurred at either one. Also, the method does not require the careful technic necessary to measure diameters with corresponding accuracy. Temperature variations do not materially affect the accuracy with the indentation method, hence it is not necessary to bring the cylinder to a standard temperature before making the measurements. This results in a saving in time. In this connection, the apparatus is provided with locating pins, and is so arranged that a mark made at given pin settings will fall within the field of vision of the periscope when using the same settings. This eliminates loss of time in searching for the marks that are too small to be readily discernable to the eye.

\section{Preliminary Experiments and Calibration}

Preliminary experiments were made to determine whether the marks would retain their shape sufficiently well to provide reasonable accuracy of measurement. A study of grinding and lapping operations using flat blocks suitably indented indicated that there was probably greater likelihood of the mark retaining its shape when the long axis of the base is perpendicular to the line of motion of the rubbing surfaces. With the lapping operations it was found that when the motion was parallel to the long axis the scratches tended to obscure the points of the mark. Subsequent trial runs in engines indicated that marks perpendicular to the axis of the cylinder were in general satisfactory for the purpose.

Accurate measurements were made of the contour of the diamond in order to determine the ratio of depth to the long axis of an indentation. It was felt, however, that greater accuracy could be obtained by direct calibration of typical indentations made in various materials. As the 
indentations are made by pressing the diamond point into the metal it would be expected that the marks would be shallower than the diamond point because of the elastic recovery of some of the metal after the stress was removed. These calibrations were obtained by comparing the changes in lengths of indentations placed on flat blocks of cylinder and piston materials with direct measurements of change in thickness occurring when material is lapped from the surfaces.

The results of these calibrations are given in figure 5 where the wear (average depth of material lapped from the surface) is plotted against the average change in length for a number of marks on a given block. The ratio of change in length

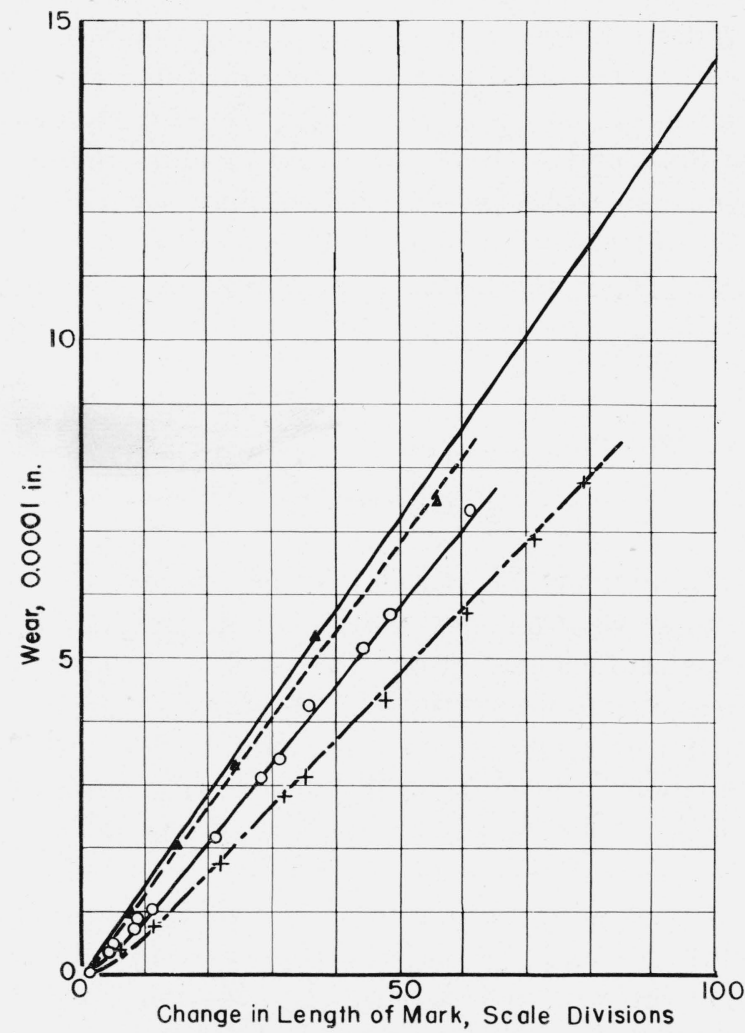

FIGURE 5.-Indentation calibration curves for various materials.

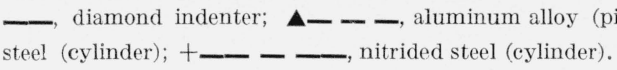

to change in depth based on measurements of the contour of the diamond is also shown in figure 5 . Comparison of the curves in this figure indicates that the deviations of the marks from the dimensions of the diamond follow the order of hardness. The curve for the relatively soft aluminum alloy piston material is the closest to the curve for the diamond, while the greatest deviation is shown for the very hard nitrided steel.

The action of impressing the marks in the surface of the metal raises a burr around the mark. The shape of the diamond is such, however, that the major portion of the burr is formed on the sides of the mark. There is no apparent burr visible to the eye at the ends of the marks, but the presence of a very slight raising of the surface at the ends may account for the slight deviations from a straight line shown by the calibration curves. In the course of the investigation it was found that most of the burr could be easily removed by rubbing lightly with fine polishing paper. For use with the cylinders, a sheet-metal guard similar to the usual erasing shield but of larger dimensions was found convenient. The holes in this guard were so arranged that the polishing paper could be rubbed with one finger over a small area at each mark on the cylinder wall.

As the marks are placed with their long axes perpendicular to the axis of the cylinder, the relation between the change in depth of mark to change in length of long axis is affected by the curvature of the cylinder. While this effect is relatively small for the particular cylinders used, suitable corrections for curvature provide greater accuracy in determining wear. The calibration curve corrected for curvature for the chrome-molybdenum steel cylinders used in most of the engine tests is shown in figure 6 . The corrections for curvature

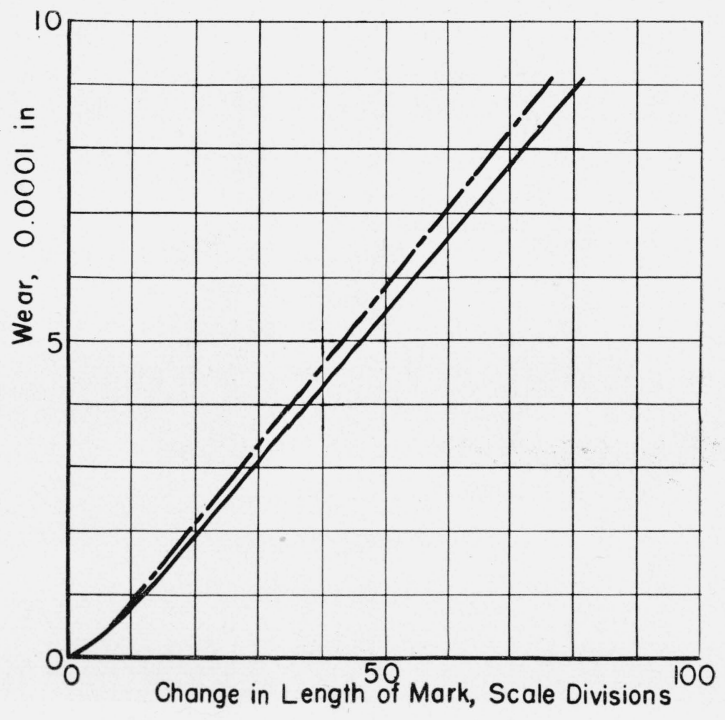

Figure 6.-Corrections for curvature of the cylinder. - - - flat block; - 
are based upon a mark having a length of 0.032 in. and upon a cylinder diameter of $5^{3 / 16}$ in., these values being representative of most test conditions.

A study was also made of the possibility of error due to the base of the diamond indenter not being parallel to the surface when the indentation is made. This would result in an unsymmetrical mark. Computations indicated that if the diamond-shaped mark was symmetrical to within a ratio not less than 41 to 50 along its major axis (ratio of dimension " $A$ " to " $B$ " in fig. 1 ), the error would be not greater than 1 percent (approximately 0.00001 in.). It was found that the apparatus could be adjusted to produce a symmetrical mark, and that other marks either in the same cylinder or in other cylinders of the same nominal size could be made well within the limits of symmetry without further adjustment. Accordingly, no corrections for lack of symmetry were necessary.

\section{Performance of Marks in Service}

One of the important considerations in this investigation was the question as to whether the marks would retain their shape during the operation of the engine. Results of a number of engine tests have shown that marks made in the cylinders of radial aircraft engines retain their shape sufficiently to provide reasonably accurate wear measurements. Photographs of marks in cylinders that were taken after the engines had been operated are shown in figures 7 and 8 . The views in figure 7 are typical of most of the marks observed. In a few cases the marks were distorted to the extent shown by the views in figure 8 . The righthand view in this figure is unusual in that the metal has flowed over part of the mark. Experience has shown that most of the poor marks occur when they have been worn to about one quarter of their original length or less, hence greater accuracy may be obtained by so regulating size of mark and length of test to avoid this condition.

The scales shown in the views in figures 7 and 8 represent an actual length of $1 \mathrm{~mm}$ (0.0394 in.), and the marks were originally about $0.9 \mathrm{~mm}$. When observed in the viewing apparatus the scale is about $2 \frac{1}{2}$ in. long. With the diamond used and with the chrome-molybdenum steel cylinders the total length of the scale represents about 0.0012 in. in depth, hence one of the small-scale divisions
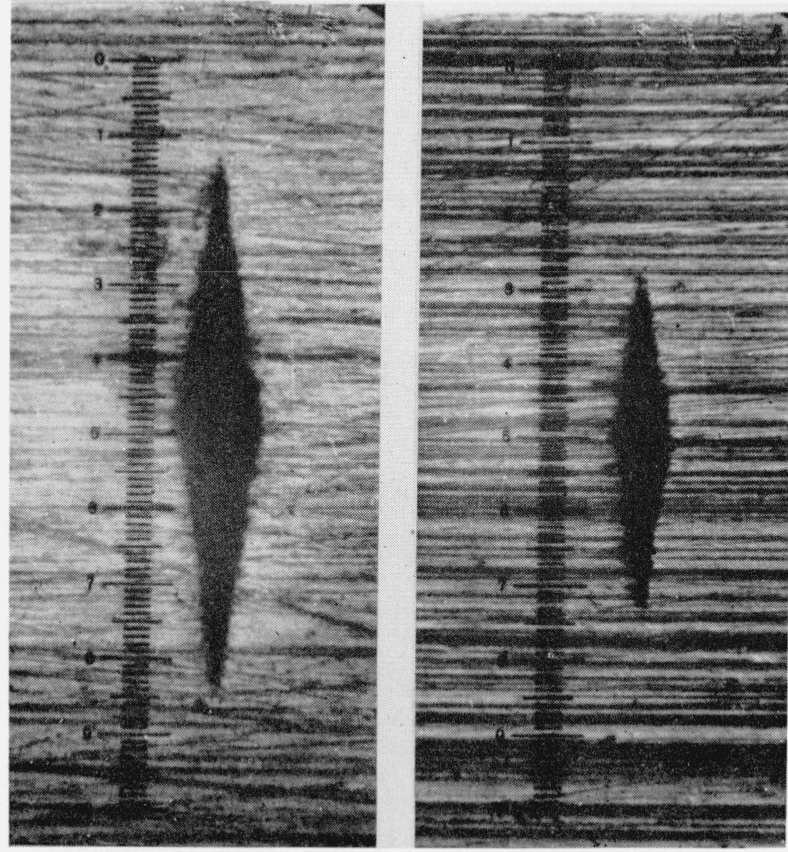

FIGURE 7.-Typical marks in cylinders after operation.
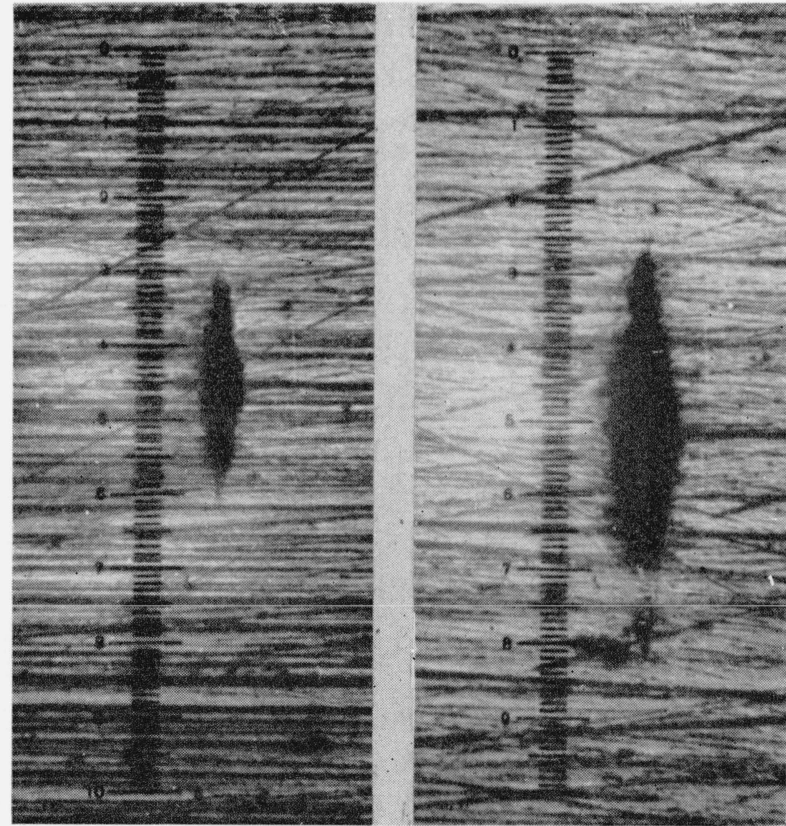

Figure 8.-Cylinder marks that were distorted by operation.

is equivalent to slightly more than $0.00001 \mathrm{in.}$ of wear. This gives an idea as to the order of sensitivity of the method.

The accuracy of the wear measurements, of course, depends upon the condition of the mark. With new marks the points are sharp and the 
lengths can be accurately determined. After the engine has been operated however, as is seen in the figures, the marks are somewhat blunted and it is necessary to estimate the position of the point. It is estimated that under normal conditions the wear can be determined with an over-all accuracy of $\pm 0.00004 \mathrm{in}$. Under favorable conditions this figure can be reduced to \pm 0.00002 in. This estimate applies to the determinations of cylinder wear. Operating experience with marks in the pistons indicates that with the softer aluminum alloy the marks have a much greater tendency to distort, and the results are not especially satisfactory. Photographs of typical marks in pistons after operation are given in figure 9. Another

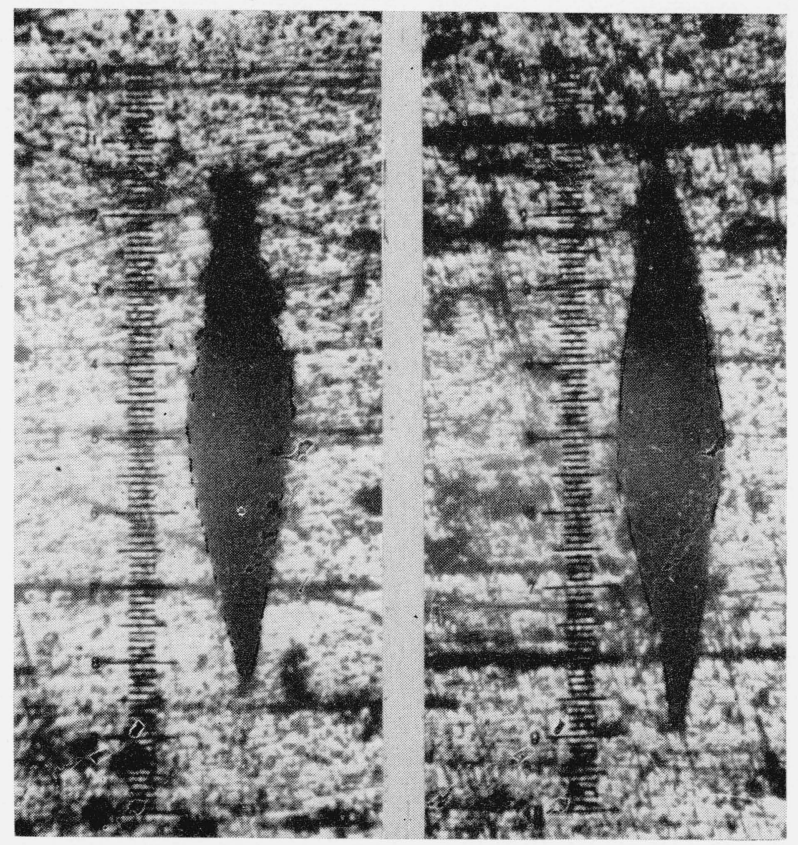

FIGURE 9.-Marks in aluminum pistons after operation.

difficulty with this material is the tendency for it to become impregnated with carbon, making it difficult to see the marks.

A second consideration of importance in this investigation was whether the presence of the marks materially affected the lubrication in the cylinder. Apparently they had little effect. This is illustrated by the views in figures 7 and 8 where it is seen that the surface scratches and ridges passing through a mark are not materially different from those beyond the ends of the mark. In this connection, the viewing apparatus provides a convenient means for the microscopic examination of the surface conditions in the cylinders.

\section{Typical Wear Patterns in Engine Cylinders}

Most of the engine tests for the study of the performance of the marks in service were made with radial aircraft engines having chromemolybdenum steel cylinder barrels $5 \% 16$ in. in diameter. In these tests 24 marks were made in each cylinder, 6 spaced 60 degrees apart at each of 4 levels. These levels were approximately 2,4 , 6 and 8 in. respectively from the open-skirt end of the cylinder. The upper row of marks was near the top of the ring travel and was in contact with the top ring only while the lower row was contacted by the oil ring only.

Typical cylinder-wear patterns obtained in these tests are shown in figures 10,11 and 12 . In these figures the wear at each of the four levels is plotted as ordinates and the angular position of the marks as abscissas. The angular locations are taken in a clockwise direction when looking into the open end of the cylinder. Zero (0)
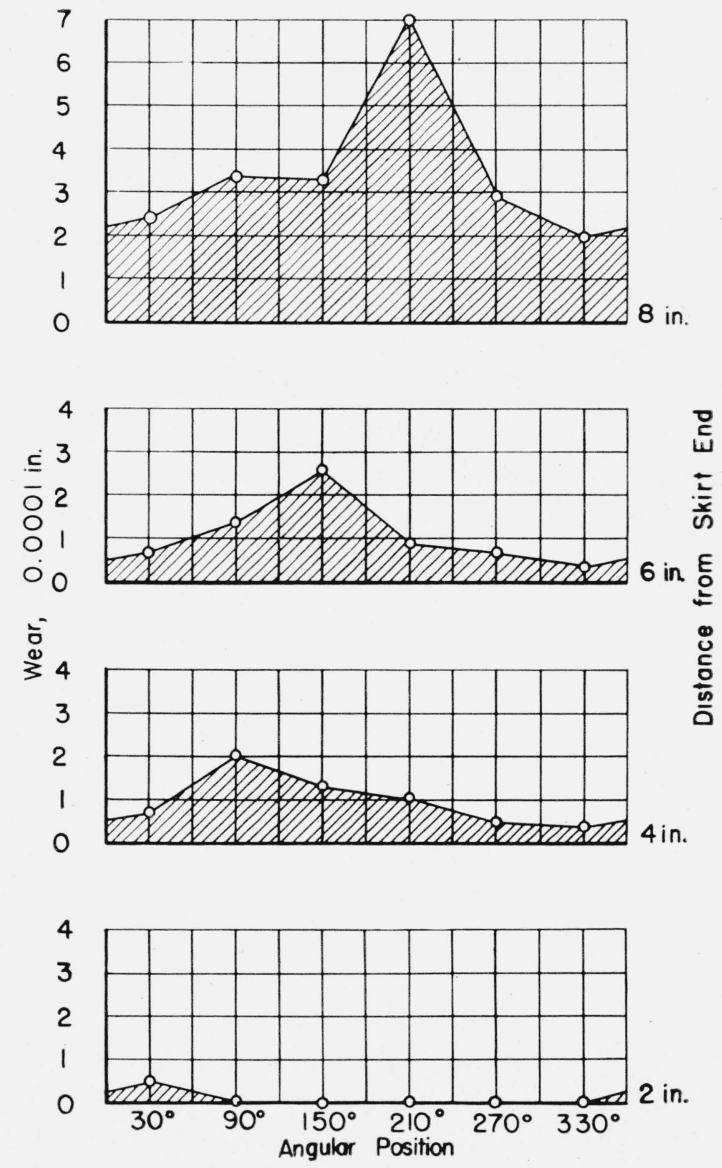

Figure 10.-Wear in cylinder of radial aircraft engine. 

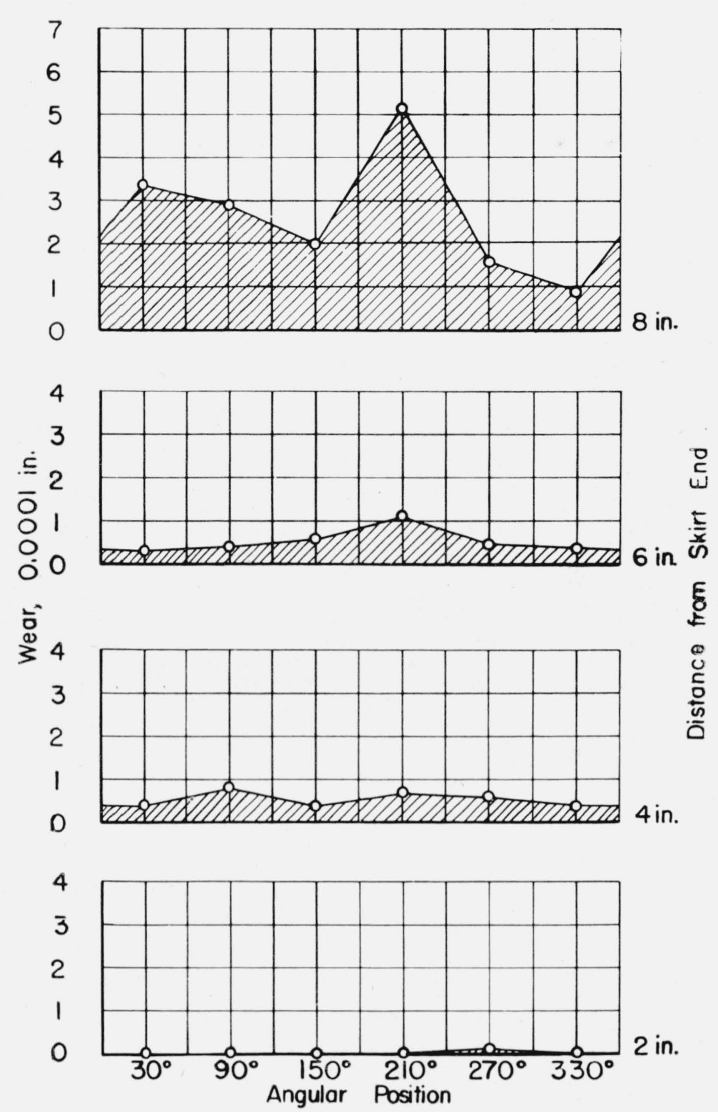

FiguRE 11.-Wear in cylinder of radial aircraft engine.

degrees is at the center line of the spark-plug hole located between the rocker-arm cages.

These figures are of interest in that they indicate an unsymmetrical wear with respect to the axis of the cylinder that would be impossible to determine with the usual measurements of diameter.

\section{Conclusion}

The data given in this paper indicate that the indentation method is practicable for the measurement of wear in radial aircraft engine cylinders. It is apparent that the method is also applicable for use on other machine elements, gages, dies, and other wearing surfaces where the material is of such a nature that the marks will retain their shape in service.

The method is covered by U. S. Patent No. 2,233,403, granted to H. C. Dickinson and S. A. McKee on March 4, 1941, and assigned by them to the United States Government. The American Instrument Company, Silver Spring, Md. was licensed to manufacture the instrument by the
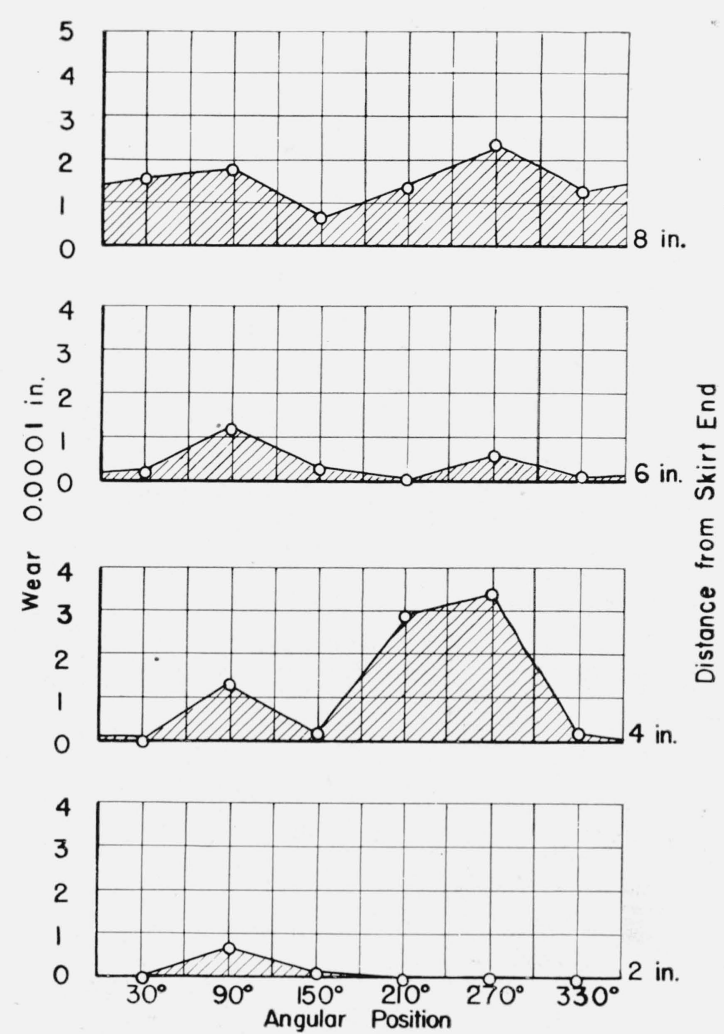

Figure 12.-Wear in cylinder of radial aircraft engine.

Secretary of Commerce. This company has developed a modification of the instrument for use with automobile engines under the trade name "Mckee Wear Gage"."2

The indentations used in this investigation were made by impressing into the test surface the apex of a four-sided diamond pyramid, which when viewed through the microscope was in the form of a sharp-pointed diamond. The chief limitation from the standpoint of accuracy was that the sharp points of the marks were blunted to some extent when wear occurred. A promising field for further work is to investigate the possibility of the use of cutting or grinding methods for producing marks without burrs and without sharppointed ends.

Acknowledgment is made to H. C. Dickinson, who instigated this investigation, and who, with C. E. Haven, F. A. Case, the late F. C. Knoop and other members of the Bureau staff, assisted in the development of the method.

Washington, May 5, 1947.

${ }^{2}$ Aminco-Mckee Wear Gage, catalog 45, p. 208, American Instrument Co., Silver Spring, Md. 